\title{
Características físicas, reológicas e sensorial de produtos extrusados de misturas de farinha de maracujá e fécula de mandioca
}

\author{
Physical, rheological and sensory characteristics of extruded products \\ from mixtures of passion fruit flour and cassava starch
}

\author{
Fernanda Rossi Moretti Trombini ${ }^{\mathrm{I}}$ Magali Leonel $^{\mathrm{II}}$ Martha Maria Mischan ${ }^{\mathrm{III}}$
}

\section{RESUMO}

Este trabalho teve por objetivo avaliar o efeito da temperatura de extrusão e da rotação da rosca sobre as propriedades físicas, reológicas e sensoriais de snacks de mandioca e maracujá produzidos em extrusor mono-rosca. Foi utilizado o delineamento central composto rotacional, com 11 tratamentos, considerando como variáveis dependentes: índice de expansão (IE), volume específico (VE), índice de solubilidade em água (ISA), índice de absorção em água (IAA), cor ( $\left.L^{*}, a^{*}, b^{*}\right)$ e propriedades de pasta (RVA). Os produtos foram aromatizados $e$ analisados para a aceitação global. Os resultados obtidos demonstraram efeitos significativos da temperatura e da rotação sobre as propriedades estudadas e boa aceitação dos produtos sabor chocolate.

Palavras-chave: amido, fibra, viscosidade, expansão, aceitabilidade.

\section{ABSTRACT}

This research had as objective to evaluate the effect of extrusion temperature and screw speed on physical and rheological properties, as well as, the sensory acceptance of cassava and passion fruit snacks produced in single-screw extruder. A central composite design with 11 treatments was used, considering as dependent variables: expansion index (EI), specific volume (SV), water solubility index (WSI), water absorption index (WAI), color ( $\left.L^{*}, a^{*}, b^{*}\right)$ and pasting properties (RVA). The products were flavored and analyzed for global acceptance. The results showed significant effects of temperature and screw speed on the dependent variables. The chocolate flavored snacks obtained good acceptance.

Key words: starch, fiber, viscosity, expansion, acceptance.

\section{INTRODUÇÃO}

Uma tecnologia que tem sido muito utilizada pelas indústrias no desenvolvimento de produtos alimentícios é a extrusão. Nesse processo, a matéria-prima é cozida e texturizada por ação combinada da umidade, pressão, temperatura e cisalhamento mecânico, levando à formação de uma nova estrutura molecular e gerando uma série de produtos prontos para o consumo, como snacks e cereais matinais ou ingredientes com largo emprego na indústria de alimentos (SINGH et al., 2007).

Uma possibilidade de melhorar produtos extrusados poderia ser a utilização de subprodutos agroindustriais, como é o caso das cascas do maracujá, que correspondem a cerca de $60-70 \%$ do peso total do fruto. A farinha de casca de maracujá é obtida após processos de trituração, desidratação e moagem, podendo ser considerada uma fonte interessante de fibra alimentar (CORDOVA et al., 2005). A composição química das cascas do maracujá desidratadas (base seca) revela elevado teor de fibra alimentar (60-70\%), sendo fonte atrativa de pectina (20-27\%), baixo teor de extrato etéreo $(0,4-0,6 \%)$ e teores consideráveis de proteína $(5-7 \%)$ e matéria mineral (6-7\%) (MATSUURA, 2005).

$\mathrm{O}$ uso da fécula de mandioca com fonte de amido em misturas para extrusão confere ao produto

IPrograma de Pós-graduação em Energia na Agricultura, Faculdade de Ciências Agronômicas, (FCA) Universidade Estadual Paulista Júlio de Mesquita Filho (UNESP), Botucatu, SP, Brasil.

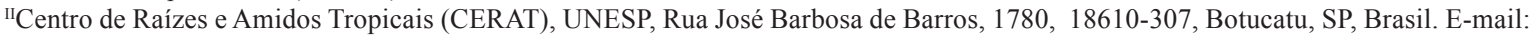
mleonel@cerat.unesp.br. Autor para correspondência.

IIIDepartamento de Bioestatística, Instituto de Biociências (IB), UNESP, Distrito de Rubião Júnior, Botucatu, SP, Brasil. 
alta expansão, leveza e crocância. Contudo, apesar de suas características químicas, físicas e reológicas, as quais permitem seu uso em diversos processos industriais, a fécula possui baixo teor de proteínas e fibras, nutrientes de interesse para o desenvolvimento de snacks com maior valor nutricional (RICKARD et al., 1991).

No desenvolvimento de novos produtos, é imprescindível aperfeiçoar parâmetros, como forma, cor, aparência, odor, sabor, textura, consistência e a interação dos diferentes componentes, com a finalidade de alcançar um equilíbrio que se traduza em qualidade e aceitabilidade (BARBOZA et al., 2003).

As variáveis do processo de extrusão que controlam diretamente os atributos de qualidade do produto são chamadas variáveis independentes. As mais importantes são a composição dos ingredientes alimentares, sua umidade, o tamanho das partículas, a velocidade de alimentação do parafuso, a configuração do parafuso e da matriz, a temperatura, a pressão e o tempo de residência do alimento no cilindro extrusor. Já as variáveis dependentes mudam como consequência das variáveis independentes e, entre elas, pode-se citar a densidade; o volume específico; a expansão; a textura; a viscosidade; os índices de absorção e de solubilidade em água, entre outros (MEUSER, 1994).

Neste trabalho, objetivou-se avaliar os efeitos da temperatura de extrusão e da rotação da rosca sobre as propriedades físicas, reológicas e sensoriais de snacks produzidos a partir de misturas de farinha de maracujá e fécula de mandioca.

\section{MATERIAL E MÉTODOS}

Foram utilizadas como matérias-primas a farinha de maracujá comercial (Jasmim), e fécula de mandioca fornecida pela empresa Halotek-Fadel $\mathrm{S} / \mathrm{A}$. As matérias-primas foram caracterizadas quanto à composição química. $\mathrm{O}$ conteúdo de umidade foi determinado de acordo com o método 44-15A, teor de nitrogênio total, pelo método $\mathrm{n}^{\circ} 46-13$, cinzas analisado pelo método $\mathrm{n}^{\mathrm{o}}$ 08-12 AACC, (2003). O teor de lipídios foi determinado de acordo com o método $\mathrm{n}^{\mathrm{o}}$ 31.4.02 (AOAC, 2005). Os carboidratos totais foram calculados por diferença (100g - gramas totais de umidade, proteína, lipídios e cinzas), incluindo a fração fibra alimentar.

Após ensaios preliminares, foram preparadas misturas contendo $10 \%$ de farinha de maracujá e $90 \%$ de fécula de mandioca (base úmida), totalizando $600 \mathrm{~g}$ de mistura por tratamento. O processamento foi realizado em linha completa de extrusão INBRA RX50, da Inbramaq S/A (Ribeirão Preto, Brasil).
O delineamento experimental utilizado foi o central, composto rotacional para dois fatores, totalizando 11 tratamentos: 4 tratamentos correspondentes ao fatorial $2^{2}$, em que os dois fatores são: $\mathrm{R}=$ rotação da rosca (rpm) e $\mathrm{T}=$ temperatura na $3^{\text {a }}$ zona de aquecimento do canhão de extrusão $\left({ }^{\circ} \mathrm{C}\right)$, cada qual em dois níveis, codificados como -1 e $+1 ; 4$ tratamentos com níveis mínimo e máximo de cada fator, codificados como $-\alpha \mathrm{e}+\alpha$, respectivamente, sendo $\alpha=$ 1,41 ; um tratamento central repetido três vezes, onde os fatores estão todos em um nível médio, codificado como zero. Dessa forma, a rotação da rosca variou de $200(-\alpha)$ a $270 \mathrm{rpm}(+-\alpha)$ e a temperatura de extrusão de $70(-\alpha)$ a $130^{\circ} \mathrm{C}(+-\alpha)$.

Os parâmetros fixos do processo definidos por ensaios preliminares foram: taxa de compressão da rosca $3: 1$, abertura da matriz $(4 \mathrm{~mm})$, taxa de alimentação de $180 \mathrm{~g} \mathrm{~min}^{-1}$, temperaturas na $1^{\mathrm{a}}$ zona $\left(25^{\circ} \mathrm{C}\right)$ e $2^{-}$zona $\left(45^{\circ} \mathrm{C}\right)$; e umidade da mistura $(12 \%)$.

As variáveis dependentes foram: índice de expansão, volume específico, índice de solubilidade em água, índice de absorção de água, cor e propriedades de pasta.

O índice de expansão foi determinado com a utilização de paquímetro digital e calculado pela relação entre o diâmetro central da amostra extrusada e o diâmetro da matriz (4 mm), sendo considerado o valor obtido pela média aritmética das medidas de 15 unidades aleatórias de produtos para cada tratamento (FAUBION \& HOSENEY, 1982).

Ovolume específico dos produtos expandidos foi determinado pelo método do deslocamento da massa ocupada de semente de painço em um béquer, sendo medido o excedente em uma proveta graduada.

Os produtos extrusados foram triturados e analisados quanto ao índice de solubilidade em água (ISA) e índice de absorção de água (IAA), seguindo a metodologia de ANDERSON (1969).

As amostras trituradas de cada tratamento foram analisadas em colorímetro Minolta CR-400 para a cor, tendo seus valores expressos em $\mathrm{L}^{*}$ (luminosidade ou brilho) variando do preto (0) ao branco (100), chroma $\mathrm{a}^{*}$ do verde $(-60)$ ao vermelho $(+60)$ e os valores do chroma b* do azul (-60) ao amarelo (+60).

As propriedades de pasta dos extrusados triturados foram avaliadas em Rapid Visco Analyser (RVA) com programação do Software Termocline for Window do RVA (Extrusion 1) (NEWPORT SCIENTIFIC, 1998).

Após a análise dos dados, realizou-se a aromatização dos produtos. Para tal aromatização, foi elaborada uma solução contendo $500 \mathrm{~g}$ de açúcar refinado (União), 100g de chocolate em pó 
(Nestlé), 50g de leite em pó (Ninho), 20mL de óleo canola (Liza) e $150 \mathrm{~mL}$ de água. Após a mistura dos ingredientes, a solução foi aquecida até a temperatura de fervura e permaneceu sob agitação para a redução de aproximadamente $50 \%$ do volume inicial.

O processo de aromatização foi realizado em drageador (Inbramaq, Ribeirão Preto, Brasil) por meio da aspersão da solução aromatizante sobre os produtos, com agitação e secagem por período de dois minutos para cada $100 \mathrm{~g}$ de produto.

Após o resfriamento, os produtos foram embalados em sacos de polipropileno codificados com três números aleatórios e avaliados sensorialmente. Cinquenta provadores não treinados consentiram em participar do estudo e avaliaram a aceitação global dos snacks em uma escala hedônica estruturada de nove pontos: 1 - desgostei muitíssimo, 5 - não gostei/nem desgostei, 9 - gostei muitíssimo (STONE \& SIDEL, 1985)

A análise dos dados foi realizada pelo programa SAS. O ajuste do modelo foi feito pela opção stepwise, sendo que o modelo obtido foi validado pelo teste F, utilizando-se como denominador o quadrado médio do erro puro (BARROS NETO, 2007).

\section{RESULTADOS E DISCUSSÃO}

A composição físico-química da farinha de maracujá mostrou $10,2 \%$ de umidade, elevado teor de fibras $(26,3 \%)$ e carboidratos totais $(49,24 \%)$, considerável teor de proteína $(7,90 \%)$ e cinzas
$(6,36 \%)$ e uma baixa concentração de matéria graxa (0,02\%). VERNAZA et al. (2009), estudando o efeito da extrusão no desenvolvimento de cereal matinal de farelo integral de maracujá obtiveram resultados próximos deste trabalho para o teor de proteínas $(7,63 \%)$ e cinzas $(6,17 \%)$.

Para a fécula de mandioca, a umidade foi de $13,1 \%$ e o componente principal foram os carboidratos totais $(82,64 \%)$. Os demais componentes mostraram teores reduzidos com $1,44 \%$ de cinzas, $0,21 \%$ de proteínas e $0,61 \%$ de matéria graxa.

Os resultados para índice de expansão variaram de 2,53 a 3,51 e o volume específico (VE) dos produtos variou de 5,58 a $8,51 \mathrm{~mL}$. $\mathrm{g}^{-1}$. Segundo o modelo estatístico, não houve efeito significativo da temperatura e da velocidade de rotação para as variáveis IE e VE (Tabela 1). Contudo, os maiores valores observados para esses parâmetros foram nas condições do ponto central, ou seja, $100^{\circ} \mathrm{C}$ de temperatura de extrusão e rotação de $235 \mathrm{rpm}$.

A expansão e volume são relacionados com a gelatinização do amido. $\mathrm{O}$ aumento na gelatinização aumenta a expansão e diminui a densidade (BHATTACHARYA \& CHOUDHURY, 1994).

LEONEL et al. (2010), estudando os efeitos de parâmetros de extrusão nas propriedades físicas de snacks de fécula de mandioca e polpa de laranja, observaram IE variando de 1,93 a 2,99 e VE de 1,87 a 8,44 , valores inferiores aos obtidos neste trabalho.

Tabela 1 - Coeficientes de regressão estimados (modelo $y_{k}=\beta_{0}+\beta_{1} x_{1}+\beta_{2} x_{2}+\beta_{12} x_{1} x_{2}+\varepsilon$ ) para índice de expansão, volume específico e parâmetros de cor dos produtos expandidos.

\begin{tabular}{llllll}
\hline \multicolumn{1}{c}{ Parâmetros } & IE & VE & L* & $\mathrm{a}^{*}$ & $\mathrm{~b}^{*}$ \\
\hline$\beta_{0}$ (intercepto) & 3,02 & 7,286 & 67,58 & 5,55 & 22,01 \\
$\beta_{1}(\mathrm{~T})$ & $-0,079 \mathrm{~ns}$ & $0,33 \mathrm{~ns}$ & $1,38^{*}$ & $-0,39 * *$ & $-0,514^{* *}$ \\
$\beta_{2}(\mathrm{RR})$ & $-0,082 \mathrm{~ns}$ & $0,421 \mathrm{~ns}$ & $1,19 *$ & $-0,24 * *$ & $-0,241^{*}$ \\
$\beta_{11}\left(\mathrm{~T}^{*} \mathrm{~T}\right)$ & $0,087 \mathrm{~ns}$ & $-0,289 \mathrm{~ns}$ & $-0,10 \mathrm{~ns}$ & $0,06 \mathrm{~ns}$ & $0,078 \mathrm{~ns}$ \\
$\beta_{22}(\mathrm{RR} * \mathrm{RR})$ & $-0,06 \mathrm{~ns}$ & $-0,012 \mathrm{~ns}$ & $-0,24 \mathrm{~ns}$ & $0,04 \mathrm{~ns}$ & $-0,059 \mathrm{~ns}$ \\
$\beta_{12}(\mathrm{~T} * \mathrm{RR})$ & $-0,162 \mathrm{~ns}$ & $-0,277 \mathrm{~ns}$ & $-0,035 \mathrm{~ns}$ & $-0,095 \mathrm{~ns}$ & $-0,377^{*}$ \\
$\mathrm{R}^{2}$ & 0,4299 & 0,6434 & 0,7719 & 0,9256 & 0,9020 \\
$\mathrm{~F} \mathrm{valor}$ & 0,75 & 1,80 & 3,38 & 12,44 & 9,20 \\
$\operatorname{Pr}>\mathrm{F}$ & 0,6177 & 0,2664 & 0,1035 & 0,0076 & 0,0146 \\
\hline
\end{tabular}

$\beta_{1}(\mathrm{~T})=$ temperatura (linear); $\beta_{2}(\mathrm{RR})=$ rotação da rosca (linear); $\beta_{11}\left(\mathrm{~T}^{*} \mathrm{~T}\right)=$ temperatura (quadrático); $\beta_{2}\left(\mathrm{RR}^{2}\right)=$ rotação da rosca $(q u a d r a ́ t i c o) ;$ $\mathrm{R}^{2}=$ coeficiente de determinação; $\mathrm{ns}=$ não significativo; $*=\mathrm{P}<0,05, * *=\mathrm{P}<0,01$, $* * *=\mathrm{P}<0,001$; IE=índice de expansão; VE=volume específico; $\mathrm{L} *=$ luminosidade; $\mathrm{a} *=$ chroma $\mathrm{a} ; \mathrm{b} *=$ chroma $\mathrm{b}$. 
A cor de produtos alimentícios, principalmente dos snacks extrusados, é um dos parâmetros tecnológicos que influencia no atrativo visual e sensorial. A mudança de cor durante o processo de extrusão pode ser um indicador para avaliar a intensidade do processo em relação às mudanças químicas e nutricionais (ILO \& BERGHOFER, 1999).

A luminosidade $\left(\mathrm{L}^{*}\right)$ dos produtos extrusados variou de 63,28 a 70,61. Os dados mostraram efeito linear da temperatura e da rotação da rosca (Tabela 1). Através da superfície de resposta, observa-se que snacks mais claros podem ser obtidos em alta temperatura e rotação da rosca (Figura 1b).

LEORO (2007), avaliando o efeito dos parâmetros operacionais sobre a cor de cereal matinal extrusado a base de farinha de milho e maracujá, observou a influência do teor de farelo de maracujá e da temperatura, com $L^{*}$ variando de 44,15 a 68,26.

O chroma a* variou de 4,79 a 6,35, com efeito significativo linear para a temperatura e a rotação da rosca (Figura 1c). Para o chroma b*, observou-se uma variação de 20,76 a 23,12, com efeito significativo linear para temperatura e rotação da rosca, bem como a interação dessas duas variáveis (Figura 1d). As superfícies de respostas traçadas a partir dos modelos ajustados mostraram que o aumento da cromaticidade ocorre com a diminuição da temperatura de extrusão e rotação da rosca (Figuras 1c e 1d).

As condições de processamento utilizadas na extrusão (alta temperatura e baixa umidade) são reconhecidas por favorecer a reação entre açúcares redutores e aminoácidos, o que resulta na formação de compostos coloridos e redução do aminoácido lisina (ILO \& BERGHOFER, 1999).

$\mathrm{O}$ índice de solubilidade em água (ISA) é um parâmetro que avalia o grau de degradação dos grânulos de amido, e o índice de absorção de água (IAA) está relacionado com a disponibilidade dos grupos hidrofilicos $(-\mathrm{OH})$ em se ligar às moléculas de água, promovendo a formação de gel (CARVALHO et al., 2002).

Os resultados obtidos nos diferentes tratamentos de extrusão mostraram uma variação de 54,59 a 88,28\% para o índice de solubilidade em água e de 5,20 a 20,79g gel $\mathrm{g}^{-1}$ para o índice de absorção em água.

A análise dos coeficientes de regressão para o índice de solubilidade em água (ISA) mostrou influência da temperatura de extrusão (Tabela 2), com maior solubilidade nas condições de elevada temperatura de extrusão (Figura 1a).

Esse resultado está em acordo com LEONEL et al. (2010), que observaram que o índice de solubilidade em água de produtos extrusados de mistura de fécula de mandioca e polpa de laranja variou de 37,76 a $84,93 \%$, com os menores valores de ISA obtidos nas condições de baixa temperatura.

O aumento da solubilidade verificado em produtos extrusados é atribuído à dispersão das moléculas de amilose e amilopectina como consequência da gelatinização, quando as condições são mais brandas, e da formação de compostos de baixo peso molecular, quando as condições são mais drásticas (COLONNA et al., 1984).

Tabela 2 - Coeficientes de regressão estimados (modelo $y_{k}=\beta_{0}+\beta_{1} x_{1}+\beta_{2} x_{2}+\beta_{12} x_{1} x_{2+\varepsilon}$ ) para o índice de solubilidade em água, índice de absorção de água e parâmetros de viscosidade para os produtos extrusados.

\begin{tabular}{|c|c|c|c|c|c|c|c|}
\hline \multirow[t]{2}{*}{ Parâmetros } & \multirow[b]{2}{*}{ ISA } & \multirow[b]{2}{*}{ IAA } & \multicolumn{5}{|c|}{ 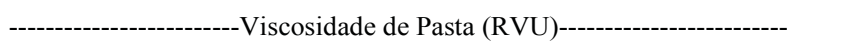 } \\
\hline & & & VI & PV & QV & VF & TR \\
\hline$\beta_{0}$ (intercepto) & 60,77 & 6,40 & 13,33 & 13,34 & 12,08 & 3,58 & 2,66 \\
\hline$\beta_{1}(\mathrm{~T})$ & $7,40 * *$ & $-0,94 n s$ & $4,29 \mathrm{~ns}$ & $3,59 \mathrm{~ns}$ & $3,59 \mathrm{~ns}$ & $0,29 \mathrm{~ns}$ & $0,29 *$ \\
\hline$\beta_{2}(\mathrm{RR})$ & $0,29 \mathrm{~ns}$ & $1,31 \mathrm{~ns}$ & $-0,77 \mathrm{~ns}$ & $-0,83 n s$ & $-0,92 \mathrm{~ns}$ & $0,009 \mathrm{~ns}$ & $-0,08 \mathrm{~ns}$ \\
\hline$\beta_{11}\left(\mathrm{~T}^{*} \mathrm{~T}\right)$ & $6,05 \mathrm{~ns}$ & $3,95 \mathrm{~ns}$ & $5,35 \mathrm{~ns}$ & $5,09 \mathrm{~ns}$ & $4,16 \mathrm{~ns}$ & $0,78 * *$ & $-0,31^{*}$ \\
\hline$\beta_{22}\left(\mathrm{RR}^{*} \mathrm{RR}\right)$ & $2,15 \mathrm{~ns}$ & $-0,12 \mathrm{~ns}$ & $1,81 \mathrm{~ns}$ & $1,24 \mathrm{~ns}$ & $0,68 \mathrm{~ns}$ & $0,66^{*}$ & $-0,07 \mathrm{~ns}$ \\
\hline$\beta_{12}\left(\mathrm{~T}^{*} \mathrm{RR}\right)$ & $3,24 \mathrm{~ns}$ & $2,28 \mathrm{~ns}$ & $0,48 \mathrm{~ns}$ & $0,56 \mathrm{~ns}$ & $0,65 \mathrm{~ns}$ & $-0,69^{*}$ & $-0,60 * *$ \\
\hline $\mathrm{R}^{2}$ & 0,7935 & 0,5203 & 0,6108 & 0,4436 & 0,3966 & 0,8556 & 0,8990 \\
\hline F valor & 3,84 & 1,08 & 1,57 & 0,80 & 0,66 & 5,92 & 8,90 \\
\hline $\operatorname{Pr}>\mathrm{F}$ & 0,0829 & 0,4655 & 0,3164 & 0,5952 & 0,6717 & 0,0366 & 0,0157 \\
\hline
\end{tabular}

$\beta_{1}(\mathrm{~T})=$ temperatura (linear); $\beta_{2}(\mathrm{RR})=$ rotação da rosca (linear); $\beta_{11}\left(\mathrm{~T}^{*} \mathrm{~T}\right)=$ temperatura (quadrático); $\beta_{1}\left(\mathrm{RR}^{2}\right)=$ rotação da rosca $(q u a d r a ́ t i c o) ;$ $\mathrm{R}^{2}=$ coeficiente de determinação; ns $=$ não significativo; $*=\mathrm{P}<0,05, * *=\mathrm{P}<0,01, * * *=\mathrm{P}<0,001$; ISA=índice de solubilidade em água $(\%)$; IAA=índice de absorção de água; $\mathrm{VI}=$ viscosidade inicial; $\mathrm{PV}=$ pico de viscosidade; $\mathrm{QV}=$ quebra de viscosidade; $\mathrm{VF}=\mathrm{viscosidade}$ final; $\mathrm{TR}=$ tendência à retrogradação. 
Para o índice de absorção de água (IAA), não foi observado efeito significativo dos fatores temperatura e rotação da rosca, conforme mostra a tabela 2. Contudo, os menores IAA foram observados em temperaturas intermediárias de extrusão $\left(105^{\circ} \mathrm{C}\right)$.

A extrusão provoca alterações em várias propriedades funcionais do amido, as quais dependem principalmente da relação amilose e amilopectina e dos parâmetros operacionais do processo (FELLOWS, 2000).

As propriedades de pasta dos produtos extrusados mostraram que a viscosidade inicial variou de 12,67 a $38,17 \mathrm{RVU}$, o pico de viscosidade de 12,08 a 38,58 RVU, e a quebra de viscosidade de 9,17 a 35,42RVU, não ocorrendo efeitos significativos da temperatura e rotação da rosca (Tabela 2). Os maiores

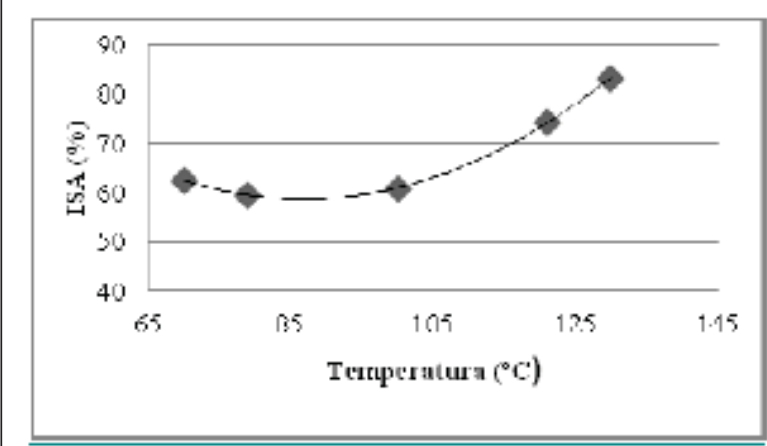

(a)

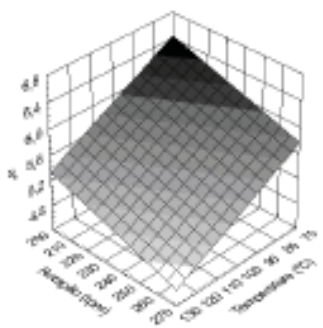

(c)

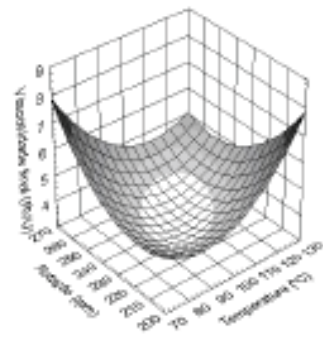

(e)

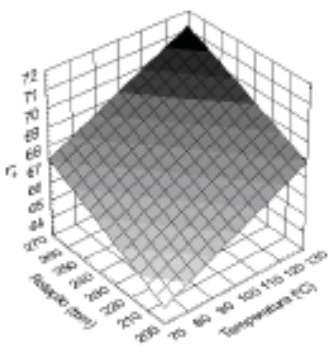

(b)

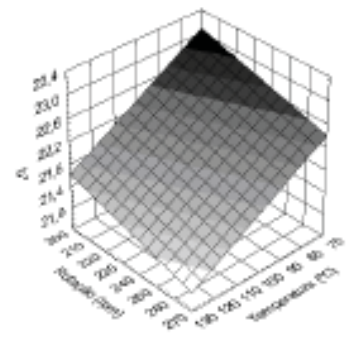

(d)

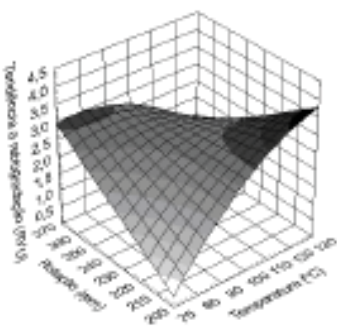

(f)

Figura 1 - Efeitos de parâmetro de extrusão sobre o índice de solubilidade em água (a), luminosidade (b), chroma a* (c) chroma b* (d), viscosidade inicial (e), tendência à retrogradação (f). 
valores observados para esses parâmetros foram na condição de $130^{\circ} \mathrm{C}$ de temperatura de extrusão e $235 \mathrm{rpm}$.

Na viscosidade final, os resultados variaram de 3,17 a 6,25RVU e a tendência a retrogradação variou de 1,42 a 3,33RVU. Os coeficientes de regressão mostraram efeitos quadráticos da temperatura e da rotação da rosca, bem como da interação dos fatores sobre a viscosidade final das misturas extrusadas (Tabela 2). Nas condições extremas opostas da temperatura e rotação da rosca, são obtidas as maiores viscosidades finais das misturas. Já nas condições centrais dos fatores, observaram-se as menores viscosidades finais (Figura 1e).

Para a tendência a retrogradação, ocorreu efeito linear e quadrático da temperatura de extrusão, bem como, efeito da interação da temperatura com a rotação (Tabela 2). O gráfico de superfície de resposta, traçado a partir do modelo ajustado, mostrou que as maiores tendências à retrogradação são observadas nas condições extremas opostas de temperatura e rotação da rosca (Figura 1f).

LUSTOSA et al. (2009), estudando as propriedades de pasta de farinha de mandioca extrusada, encontraram valores superiores para a VF (8,83 a 28,17RVU) e TR (4,91 a 15,75RVU). De acordo com os autores, a baixa viscosidade final e a baixa tendência a retrogradação podem indicar severidade dos tratamentos, com degradação dos polímeros do amido, rompimento das estruturas moleculares e redução da capacidade de recristalização destes.

Para a aromatização e análise sensorial, selecionou-se a condição central de temperatura de extrusão e rotação da rosca, quando se obtiveram produtos com maior expansão, coloração clara, índice de absorção, índice de solubilidade em água e propriedades de pasta com valores intermediários, características desejadas para esse tipo de produto. Os resultados obtidos na análise sensorial global dos produtos extrusados aromatizados evidenciaram que $46 \%$ dos provadores gostaram regularmente (valor 7), 20\% dos provadores gostaram muito (valor 8), $6 \%$ dos provadores (gostaram extremamente (valor 9), 24\% dos provadores (gostaram ligeiramente (valor 6), e 4\% dos provadores (desgostaram ligeiramente (valor 4). Sendo assim, os snacks de fécula de mandioca com farinha da casca de maracujá, processados à temperatura de $100^{\circ} \mathrm{C}$ e $235 \mathrm{rpm}$ e aromatizados com calda de chocolate tiveram aprovação de $72 \%$.

\section{CONCLUSÃO}

Os resultados obtidos mostram que a farinha de maracujá é um produto com considerável teor de fibra e proteína, podendo ser misturado à fécula de mandioca para a obtenção de produtos extrusados. As condições intermediárias de temperatura $\left(100^{\circ} \mathrm{C}\right)$ e rotação $(235 \mathrm{rpm})$ testadas foram adequadas para a obtenção de produtos com características tecnológicas desejadas e boa aceitação global após aromatização.

\section{REFERÊNCIAS}

AMERICAN ASSOCIATION OF CEREAL CHEMISTRY. Approved methods of the American Association of Cereal Chemists. 10.ed. rev. St. Paul, 2003. s. n. p.

ANDERSON, R.A. Gelatinization of corn grits by roll and extrusion cooking. Cereal Science Today, v.14, n.1., p.4-11, 1969. Disponível em: <http://onlinelibrary.wiley.com/doi/10.1002/star. v22:4/issuetoc>. Acesso em: 03 maio, 2012.

ASSOCIATION OF OFFICIAL AGRICULTURAL CHEMISTRY. Official Methods of Analysis. 18.ed. Washington, DC, 2005. 2598p.

BARBOZA, L.M.V. et al. Desenvolvimento de produtos e análise sensorial. Brasil alimentos, n. 18, p.34-35, 2003. Disponível em: <http:// www.signuseditora.com.br/ba/pdf/18/18\%20-020Desenvolvimento. pdf $>$. Acesso em: 10 set. 2012.

BARROS NETO, B. et al. Como fazer experimentos: pesquisa e desenvolvimento na ciência e na indústria. 3.ed. Campinas: Unicamp, 2007. 480p.

BHATTACHARYA, S.; CHOUDHURY, G.S. Twin-screw extrusion of rice flour: effect of extruder length-to-diameter ratio and barrel temperature on extrusion parameters and product characteristics. Journal of Food Process and Preservation, v.18, p.389-406, 1994. Disponível em: <http://onlinelibrary.wiley.com/ doi/10.1111/j.1745-4549.1994.tb00261.x>. Acesso em: 15 ago. 2012.

CARVALHO, R.V. et al. Efeito dos parâmetros de extrusão nas propriedades físicas de extrusados $(3 \mathrm{G})$ de misturas de farinhas de trigo, arroz e banana. Ciência e Agrotecnologia, Lavras, v.26, n.5, p.1006-1018, 2002.

COLONNA, P. et al. Extrusion cooking and drum drying of wheat starch. I. Physical and macromolecular modifications. Cereal Chemistry, v.61, n.6, p.538-543, 1984. Disponivel em: <http:// www.aaccnet.org/publications/cc/backissues/1984/Documents/ Chem61_538.pdf>. Acesso em: 2 abr. 2011.

CORDOVA, K.R.V. et al. Características físico-químicas da casca do maracujá-amarelo (Passiflora edulis Flavicarpa Degener) obtida por secagem. Boletim do CEPPA, v.23, n.2, p.221-230, 2005. Disponível em: <http://ojs.c3sl.ufpr.br/ojs2/index.php/ alimentos/article/view/4491/3497>. Acesso em: 10 set. 2012.

FAUBION, J.M.; HOSENEY, R.C. High temperature and short time; extrusion-cooking of wheat starch and flour. I. Effect of moisture and flour type on extrudate properties. Cereal Chemistry, v.59, n.6, p.529533, 1982. Disponível em: <http://www.aaccnet.org/cerealchemistry/ backissues/1982/chem59_529.pdf>. Acesso em: 10 abr. 2011.

FELLOWS, P.J. Food processing technology: principles and practice. 2.ed. Cambridge: Midway Technology, 2000. 562p. 
ILO, S.; BERGHOFER, E. Kinetics of colour changes during extrusion cooking of maize grits. Journal of Food Engineering v.39, n.1, p.73-80, 1999. Disponível em: <http://www. sciencedirect.com/science/article/pii/S0260877498001484>. Acesso em: 2 abr. 2012.

LEONEL, M. et al. Produção de snacks extrusados à base de polvilho doce e fibra de laranja. Ciência Rural, v.40, n.6, p.14111417, 2010. Disponível em: <http://www.scielo.br/pdf/cr/v40n6/ a621cr3028.pdf>. Acesso em: 10 abr. 2011. doi: 0103-84-78.

LEORO, M.G.V. Desenvolvimento de cereal matinal extrusado orgânico à base de farinha de milho e farelo de maracujá 2007. 147f. Dissertação (Mestrado em Tecnologia de Alimentos)Universidade Estadual de Campinas, SP.

LUSTOSA, B.H.B. et al. Produção de farinha instantânea de mandioca: efeito das condições de extrusão sobre as propriedades térmicas e de pasta. Acta Scientiarum Technology, v.31, n.2 v.2, p.231-238, 2009. Disponível em: <http://periodicos.uem.br/ojs/ index.php/ActaSciTechnol/article/view/892/892>. Acesso em: 2 abr. 2011.

MATSUURA, F.C.A.U. Estudo do albedo de maracujá e de seu aproveitamento em barra de cereais. 2005. $138 \mathrm{f}$. Tese (Doutorado em Tecnologia de Alimentos) - Faculdade de Engenharia de Alimentos, Universidade Estadual de Campinas, SP.
MEUSER, F. Wheat utilization for the production of starch, gluten and extruded products. In: BUSHUK, W.; RASPER, V.F. (Ed.). Wheat: production, properties, quality. London: Chapman \& Hall, 1994. Cap.13, p.179-204.

NEWPORT SCIENTIFIC. Operation manual for series 4: Instrutions manual. Warriewood, Austrália, 1998. 123p.

RICKARD, J.E. et al. The physicochemical properties of cassava starch. Tropical Science, v.31, p.189-207, 1991.

SINGH, S. et al. Nutritional aspects of food extrusion: a review. International Journal of Food Science \& Technology, v.42, n.8, p.916-929, 2007. Disponível em: <http://ac.els-cdn.com/ S0308814605008307/1-s2.0-S0308814605008307-main.pdf? tid $=7 \mathrm{c} 81156 \mathrm{~d} 711315 \mathrm{cdb} 65 \mathrm{~b} 146629 \mathrm{cb} 4 \mathrm{c} 9 \mathrm{e} \& \mathrm{acdnat}=133278715 \overline{6} \quad 1$ 1878fe217d5f34ecb3923da040d4a67>. Acesso em: 2 abr. $201 \overline{1}$. doi:10.1111/j.1365-2621.2006.01309.x.

STONE, H.; SIDEL, J.L. Sensory evaluation practices. London: Academic, 1985. 311p.

VERNAZA, M.G. Efeito do teor de farelo de maracujá e da umidade e temperatura de extrusão no desenvolvimento de cereal matinal funcional orgânico. Brazilian Journal of Food Technology. v.12, n.2, p.145-154, 2009. Disponível em: <http://bj.ital.sp.gov.br/artigos/ html/busca/PDF/v12n2374a.pdf>. Acesso em: 14 maio, 2011. 